\title{
Quality and reliability of the information on YouTube Videos about Botox injection on spasticity
}

\section{Aysel Gürcan Atci}

\author{
Istanbul Baltalimanı Bone and Joint Research and Education Hospital, \\ Physical Medicine and Rehabilitation Department, TURKEY
}

\begin{abstract}
Background: This study analyzes the botox injection on spastisite videos that have the highest views and likes on YouTube, and attempts to reveal the video qualities in order to contribute to the literature.

Methods: For review, "botox injection on spastisite" was written to the standard YouTube search bar, and the videos with the highest views were ranked using advanced search preferences. The 69 most widely viewed videos were watched and scored by one physician.

Results: The mean Modified DISCERN Score of the videos was 2,66+/-1,032 (the lowest: 1; the highest: 4) while the mean GQS score was 2,876+/-1,06 (the lowest: 1; the highest:4). In addition, the mean DISCERN score and the mean GQS value were 3,51 and 3,82, respectively, for the informational videos that were uploaded by health professionals but did not contain actual surgery.

Conclusion: We think that medical associations and state authorities in medicine should check the validity and accuracy of the information on the internet and should support the society in access to the most correct information.
\end{abstract}

\section{INTRODUCTION}

In recent years, the rate of receiving information from the internet has increased in almost every subject in daily practice due to the developing and increasing frequency of internet usage. Patients and health professionals apply to the Internet for information on many healthrelated issues. Among these sources of application, YouTube is the biggest video archive website in the world and attracts $95 \%$ of internet users with 30 million active users every day(1). There are also many health-related videos in the archive. Generally, patients apply to a physician and get detailed information about recommended treatments but they are also inclined to watch on YouTube the operation to be carried out. Therefore; the quality of a video, the persons who shot it and whether such video contains correct information are matters of great importance.

Spasticity is characterized by an increase in muscletone resulting from upper motor neuron lesions. It is a common condition in the upper

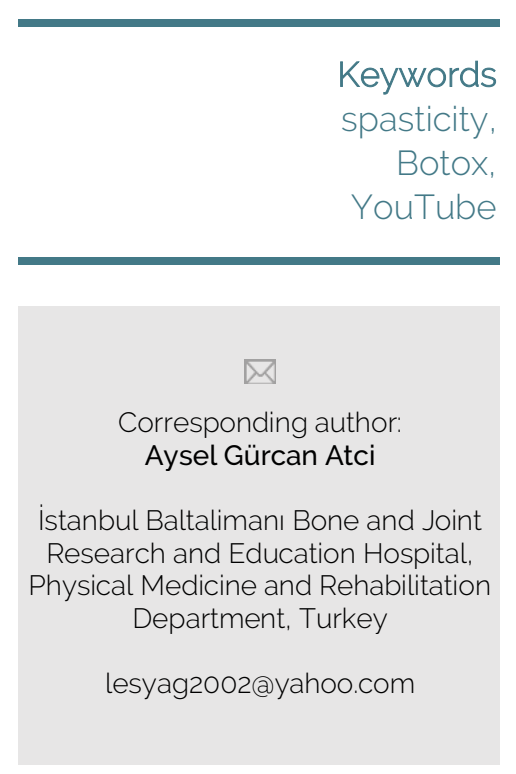

Copyright and usage. This is an Open Access article, distributed under the terms of the Creative Commons Attribution Non-Commercial No Derivatives License (https://creativecommons org/licenses/by-nc-nd/4.0/) which permits noncommercial re-use, distribution, and reproduction in any medium, provided the original work is unaltered and is properly cited.

The written permission of the Romanian Society of Neurosurgery must be obtained for commercial re-use or in order to create a derivative work.

ISSN online 2344-4959 (C) Romanian Society of Neurosurgery

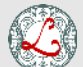

First published December 2019 by London Academic Publishing www.lapub.co.uk 
and lower extremity muscles after stroke in general cerebrovascular events (2). It is observed in $16 \%$ of patients after stroke (3). The increase in muscletone is a condition that makes mobilization and positioning difficult, delaying recovery to function that makes patients' daily life difficult. Spasticity is tried to be treated by various methods. Botulinium toxin is the most common invasive treatment method.

This study analyzes the botox injection on spastisite videos that have the highest views and likes on YouTube, and attempts to reveal the video qualities in order to contribute to the literature.

\section{MATERIAL AND METHOD}

\section{Search Strategy and Data Collection}

For review, "botox injection spastisite " was written to the standard YouTube search bar, and the videos with the highest views were ranked using advanced search preferences. The 69 most widely viewed videos were watched and scored by 1 physician.

\section{Inclusion and Exclusion Criteria}

The videos that were not in English language or did not have subtitles or speech or that did not explain the operation were eliminated.

\section{Variables Extracted}

Views, upload dates, like rates, uploaders, video lengths, comment numbers, like numbers and dislike numbers were identified as well as whether they were actual or animated videos.

Inaddition, video powerindex (VPI) values [(number of likes/number of likes $p$ number of dislikes) 100] were calculated to evaluate thepopularity of the videos.

\section{Assessment of Usefulness}

All videos were independentlye valuated by one physician for usefulness and categorized into the following mutuall exclusive categories.

1. Useful information : Videos designated as useful information were mainly focused on information delivery. They contained accurate information and were useful for learning how to do botox.

2. Misleading information :The videos contained incorrect information or did not contain usefull information.

3. Useful patient opinion: The videos in this group have the DISCERN and GQS scores as 3 or above and clearly explain the patient experiences, the performance of operations, and preoperational and postoperational pain scores.

4. Misleading patient opinion: The videos in this group have the DISCERN and GQS scores as 2 or lower and do not clearly explain patient experiences (Table 1).

\section{Scoring System}

Video reliability was scored using a modified fivepoint DISCERN tool (4), which was adapted from the original DISCERN toolforthe assessment of written healthin formation by Charnock et al(5).

The overall quality of each video was rate dusing the five-point Global QualityScale (GQS). The GQS was developed as an evaluation tool for websiteresourcesand it assesses the flowandease of use of the information presented online, and the quality of video (Table 2) [4].

Table 1: Analyses of video characteristics by usefulness category

\begin{tabular}{|l|l|l|l|l|}
\hline & $\begin{array}{l}\text { Usefullinformation } \\
(\mathrm{Gr} 1)\end{array}$ & $\begin{array}{l}\text { Misleadinginformation } \\
(\mathrm{Gr})\end{array}$ & $\begin{array}{l}\text { UsefullPatientOpinion(G } \\
\mathrm{r3})\end{array}$ & $\begin{array}{l}\text { MisleadingPatientOpinio } \\
\mathrm{n}(\mathrm{Gr} \text { ) }\end{array}$ \\
\hline Video Number & $\mathrm{n}: 11(15,9 \%)$ & $\mathrm{n}: 33(47,8 \%)$ & $\mathrm{n}: 1(2 \%)$ & $\mathrm{n}: 24(34,7 \%)$ \\
\hline Viewspeerday & $2,21+/-0,31$ & $1,257+/-0,12$ & $4,32+/-1,55$ & $3,4+/-1,3$ \\
\hline Video Lenght & $\begin{array}{l}24,562 \mathrm{~min}(2,04- \\
32,01 \mathrm{~min})\end{array}$ & $6,50 \mathrm{~min}(0,20-7,17 \mathrm{~min})$ & $12,31 \mathrm{~min}$ & $7,25 \mathrm{~min}(4,01-12,08 \mathrm{~min})$ \\
\hline Like & $22+/-14$ & $18,14+/-12,1$ & $29,08+/-2,01$ & $33,4+/-3,33$ \\
\hline Dislike & $1,32+/-0,45$ & $2,74+/-1,1$ & $1,02+/-0,32$ & $1,47+/-1,21$ \\
\hline Comments & $26,8+/-2,33$ & $27,2+/-1,33$ & $92,1+/-7,41$ & $113,4+/-11,2$ \\
\hline DiscernScore & 3,4 & 2,2 & 3,3 & 0,5 \\
\hline GQS Score & 3,9 & 2,3 & 3,8 & 1,6 \\
\hline
\end{tabular}




\section{Statistical analysis}

The results were statistically analysed using a nonparametric Kruskal-Wallis test. A p value of 0.05 or less was considered significant. The Statistical
Package for theSocial Sciences version 23 software (SPSS, Chicago, IL, USA) was used for all statistical analyses (Table 2).

\begin{tabular}{|l|l|l|l|l|l|l|}
\hline & Gr1-Gr2 & Gr1-3 & Gr1-4 & Gr2-3 & Gr2-4 & Gr3-4 \\
\hline $\begin{array}{l}\text { DiscernScore } \\
\text { p value }\end{array}$ & 0,518 & 0,708 & 0,001 & 0,652 & 0,332 & $\mathbf{0 , 0 0 1 8}$ \\
\hline $\begin{array}{l}\text { GQS Score } p \\
\text { value }\end{array}$ & 0,125 & 1,00 & $\mathbf{0 , 0 0 1}$ & 0,069 & 0,852 & $\mathbf{0 , 0 0 1}$ \\
\hline
\end{tabular}

* Values of p 0,05 was accepted

Table 2: Pairwise comparisons of video groups according to usefulness

\section{RESULTS}

69 videos with the highest views were analyzed while 31 videos were later excluded from the analysis for they were neither in English language nor contained subtitles. There were 36 technically-narrated and actual videos by professionals, 25 patient view and 8 videos were botox processing. The oldest video was uploaded in 2010 while the newest one was added to the system in 2019. The videos were uploaded by hospitals (25 videos), health professionals and physicians (20 videos), and personal accounts (21 videos) (Figure 1).

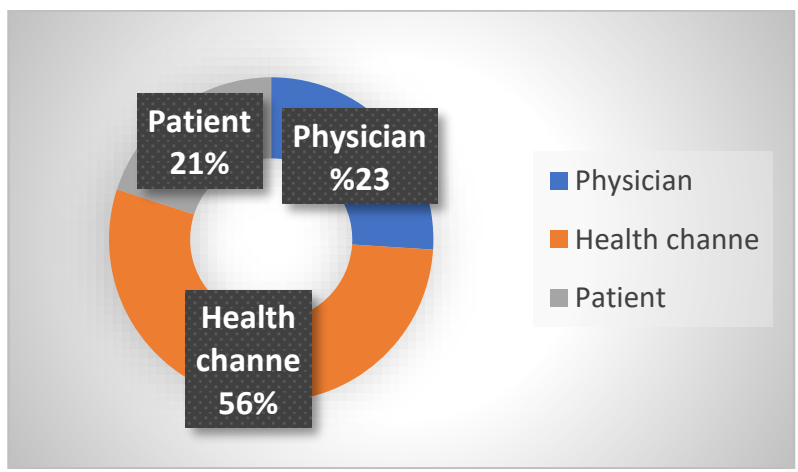

Figure 1: Uploaded videos by YouTube

The mean time of the video lengths was $41,623 \mathrm{sec}$ (the shortest: $19 \mathrm{sec}$; the longest: $54.16 \mathrm{sec}$ ), and the mean view was $88,293+/-9,75$ (the least viewed: 4075; the most viewed: 825.731). The daily mean view of the videos was $42.444+/-72,77$ (the least viewed: 5; the most viewed: 37405$)$. The mean like rate was $23.27+/-, 52$ (the most liked:360 the least liked: 0 ), and the mean dislike rate was 1,34+/-0,21 (the most disliked: 15; the least disliked: 0).As for the comments, the mean number was 4,54+/-1,23 (the least commented: 0 ; the most commented:
65).Similarly, video power index (VPI) analyses showed that the mean VPI value of the 69 videos was $0,71+/-0,14$.

The mean Modified DISCERN Score of the videos was $2,88+/-0,318$ (the lowest: 1 ; the highest: 4 ) while the mean GQS score was 3,56+/-1,206 (the lowest: 1; the highest:4) . In addition, the mean DISCERN score and the mean GQS value were 2,13 and 3,25, respectively, for the informational videos that were uploaded by health professionals. Similarly, the mean DISCERN score and the mean GQS value were 1,33 and 1,71, respectively, for the patient videos in which personal experiences were shared. No statistically significant correlation was found between the GQS and DISCERN scores according to both researchers and VPI values $(P>0.05)$.

\section{DISCUSSION}

Youtube is a video hosting site headquartered in San Bruno / California, USA. The site was founded in 2005 and started to be operated by Google in 2006. The primary purpose of the site is to download and share videos on any subject. Many health professionals, hospitals and patients share more intensive videos on medical issues. While using these videos to make inferences from the experience of the patients, the method and possible risks of the treatment to be performed by patients and their relatives; health professionals try to learn the interventional procedure live. However, there may also be incorrect, low-quality and prejudiced videos on this platform where everyone can upload videos free-ofcharge without being subject to any inspection. Pubmed reviews reveal 1089 studies that measure the quality of YouTube videos on health issues $(8,9)$. 
The first of these is a study from 2007 that evaluate the training of health professionals (7).

Botulinium toxin-related treatment applications started in 1980. It has been used in the treatment of spasticity for the last 15 years. Botulinum $A$ is a successful method used to increase theeffectiveness of pain, restlessness and physical therapy in spasticity $(10,11)$. Botulinium A toxin is the most common market name in the world as Botox $\odot$ and Dysport (c). Botox application is performed in spasticity in our country. When theliterature is screened, there are 3 controlled randomized studies on Botox $(12,13,14)$.

In our study, it was to question whether a patient with spasticity who is planned to use botox is able to get reliable information when watching the youtube video in order to obtain information and ideas before the procedure. Apart from this, it is aimed to evaluate the quality of the information that the health professionals who want to make the initiative can learn theoretically and visually from the videos. The literature review we made did not produce any study concerning the subject in question. There are various scales and measures to evaluate the quality of the information in videos and on the internet. In this study, one researcher assesses the videos using modified DISCERN scoring system, Global Quality Index and Video Power Index (VPI). According to the analysis of the 69 videos with the highest views and VPIs, it was found out that the videos presented weak and poor-quality information to patients, patient relatives and professionals who desire to learn the narrated operation. However, it was also observed that $60.9 \%$ of the videos were uploaded by health professionals and institutions. In 8 (11.5\%) videos with actual surgeries, it was seen that the average time was 15,24 seconds, the operators did not satisfactorily explain the methods before and after the operations, they did not clearly specify alternative treatments and effects and possible complications, and the videos were not supported with subtitles. It was observed that the videos did not explain the operations in simple language to convey the processes to patients and patient relatives but only the course was expressed, and that there were dialogs with patients during operations.

Furthermore, it was revealed that 11 (15.9\%) videos with the highest results of evaluation were animated or notional surgery videos, made theoretical PowerPoint presentations and were supported with anatomic cross-sections.

When the 5 most watched videos were examined, it was seen that there were 3.73 impressions per day on average and these videos belonged to hospitals installing botox application.

The videos with the highest VPI values but had 2 or below in DISCERN and GQS scoring were found to convey inadequate information. In contrast with the foregoing, the videos with the highest scores had 2,21 views every day, on average, and did not appear on the first page when searched on YouTube. However, the videos that had the highest views but contained insufficient information appeared on top in YouTube searches. Apart from these, the video comment analyses demonstrated that the highest number of comments were entered to the uploads with patient experiences. In the content of the comments, it was seen that the regression rate of complaints and the duration of the complaint-free period were examined.

Accordingly, the videos with the highest like numbers were those that contained patient remarks.

The videos were divided into 4 groups in terms of usefulness, and only 11 videos were found to contain useful and valid information. All these were uploaded by health professionals and were generally about physician remarks. The mean time of these videos was 24,562 seconds. Useful patient remarks were identified only in 1 videos, and their mean view time was 12,31 seconds.

The limitations of this study include the crosssectional design (popularity based on number of views changes constantly), and the inclusion of only the 69 most widely viewed videos (an arbitrary cut point).

\section{CONCLUSION}

As a result, it may not always be accurate to believe that the medical videos with high view, comment and like numbers on YouTube contain reliable, comprehensible and correct information. Although the access to information and videos on medical subjects is very easy in today's world, it is more appropriate to apply to experienced health professionals in order to get information. We think that medical associations and state authorities in medicine should check the validity and accuracy of the information on the internet and should support the society in access to the most correct information. 


\section{COMPLIANCE WITH ETHICAL STANDARDS}

This study does not include any human participants or animals. Videos that were available to every one were evaluated for this study. Therefore, ethics committee approval was not required.

Table 3: Discern and GQS

Modifiy Discern (1 point per question answered yes)

1. Is the video clear, concise, and understandable?

2. Are valid sources cited? (from valid studies, physiatrists or rheumatologists)

3. Is the information provided balanced and unbiased?

4. Are additional sources of information listed for patient reference?

5. Does the video address areas of controversy /uncertainty?

\section{Global quality scale}

1. Poor quality, poor flow, most information missing, not helpful for patients;

2. Generally poor, some information given but of limited use to patients;

3. Moderate quality, some important information is adequately discussed;

4. Good quality good flow, most relevant information is covered, useful for patients;

5. Excellent quality and excellent flow, very useful for patients;

\section{REFERENCES}

1. YouTube. YouTubestatisticpage. https://www. Omnicore agency.com/youtube-statistics/. Accessed October 25, 2018.

2. Lance JW. Symposium synopsis. In: Feldman RG, Young RR Koella WP, editors. Spasticity: Disordered motor control. Chicago: Year Book Medical Publishers. 1980. p. 485-94.

3. Turkel C, Sissins P. Functional activities affected by poststroke spactisity (abstract). Arch Phys Med Rehabil 2004;85:E52.
4. Singh AG, Singh S, Singh PP (2012) YouTubeforinformation on rheumatoidarthritis-a wakeupcall? J Rheumatol 39(5):899-903. https://doi.org/10.3899/jrheum.111114 (Epub 2012 Apr 1)

5. Charnock D, Shepperd S, Needham G, Gann R (1999) DISCERN: an instrumentforjudgingthequality of writtenconsumerhealthinformation on treatmentchoices. J EpidemiolCommunityHealth 53(2):105-111

6. Bernard A, Langille M, Hughes S, Rose C, Leddin D, VeldhuyzenvanZanten S (2007) A systematicreview of patientinflammatoryboweldiseaseinformationresources on the World Wide Web. Am J Gastroenterol 102(9):20702077

7. Skiba DJ (2007) NursEducPerspect. Nursingeducation 2.0: YouTube 2007 Mar-Apr;28(2):100-2.

8. Ovenden CD, Brooks FM. Anterior cervical discectomy and fusionYouTubevideos as a source of patient education. AsianSpine J. 2018;12:987-991.

9. Drozd B, Couvillon E, Suarez A. Medical YouTube videos and methods of evaluation: literature review. JMIR MedEduc. 2018;4:e3.

10. Sheean G. Botulinum toxin treatment of adult spasticity. A benefitrisk assessment. Drug Safety 2006;29:31-48.

11. Naumann M, Toyka KV, Moore P. History and current applications of botulinum toxin: from poison to remedy. In: Naumann P, Moore P, editors. Handbook of botulinum toxin treatment. 2nd ed. Cambridge (MA). Blackwell Science, 2003. p. 3-8.

12. Richardson D, Sheean G, Werring D, Desai M, Edwards S, Greenwood R, et al. Evaluating the role of botulinum toxin in adults. J Neurol Neurosurg Psychiatry 2000;69:499-506.

13. Simpson DM, Alexander DN, O'Brien CF, Tagliati M, Aswad AS, Leon JM. Botulinum toxin type $A$ in the treatment of upper extremity spasticity: a randomized, double-blind, placebo-controlled trial. Neurology 1996;46:1306-10.

14. Brashear A, Gordon MF, Elovic E, Kassicieh VD, Marciniak C, Do $M$, et al. Intramuscular injection of botulinum toxin for the treatment of wrist and finger spasticity after a stroke. N Engl J Med 2002;347:395-400 